PROCEEDINGS OF THE

AMERICAN MATHEMATICAL SOCIETY

Volume 134, Number 12, December 2006, Pages 3531-3542

S 0002-9939(06)08473-5

Article electronically published on May 31, 2006

\title{
TOEPLITZ OPERATORS ON BLOCH-TYPE SPACES
}

\author{
ZHIJIAN WU, RUHAN ZHAO, AND NINA ZORBOSKA
}

(Communicated by Joseph A. Ball)

\begin{abstract}
We characterize complex measures $\mu$ on the unit disk for which the Toeplitz operator $T_{\mu}^{\alpha}, \alpha>0$, is bounded or compact on the Bloch type spaces $B^{\alpha}$.
\end{abstract}

\section{INTRODUCTION AND PRELIMINARIES}

Let $\mathbb{D}$ be the unit disk on the complex plane. Let $d A(z)=\frac{1}{\pi} d x d y$ be the normalized Lebesgue measure on $\mathbb{D}$.

For a complex measure $\mu, \alpha>0$, and $b \in L^{1}$, define a Toeplitz operator as follows:

$$
T_{\mu}^{\alpha}(b)(z)=\alpha \int_{\mathbb{D}} \frac{\left(1-|w|^{2}\right)^{\alpha-1} b(w)}{(1-\bar{w} z)^{\alpha+1}} d \mu(w) .
$$

We also define the general Bergman projection of the measure $\mu$ and for $\alpha>-1$ as follows:

$$
P_{\alpha}(\mu)(z)=(\alpha+1) \int_{\mathbb{D}} \frac{\left(1-|w|^{2}\right)^{\alpha}}{(1-\bar{w} z)^{\alpha+2}} d \mu(w) .
$$

The general Bergman projection of the function $b$ is

$$
P_{\alpha}(b)(z)=(\alpha+1) \int_{\mathbb{D}} \frac{\left(1-|w|^{2}\right)^{\alpha} b(w)}{(1-\bar{w} z)^{\alpha+2}} d A(w) .
$$

Thus $T_{\mu}^{\alpha}(b)(z)=P_{\alpha-1}\left(\mu_{b}\right)(z)$, where $d \mu_{b}(z)=b(z) d \mu(z)$. Note that these choices of the indexes provide the standard notation for the general Bergman projections and for the standard case $\alpha=1$ as follows:

When $\alpha=1$ and the measure $\mu$ is such that $d \mu(z)=f(z) d A(z)$, with $f \in L^{1}$, we have that $T_{\mu}^{1}$ is the standard Toeplitz operator defined by

$$
T_{\mu}^{1}(b)=T_{f}(b)=P(f b), \quad \forall b \in L^{1} .
$$

Here $P=P_{0}$ denotes the standard Bergman projection. Recall that if $f$ is in $L^{\infty}$, then $T_{f}$ is bounded on the Bergman spaces $L_{a}^{p}, p>1$.

Toeplitz operators have been studied extensively on the Bergman spaces by many authors. For references, see for example [9]. In this paper we study the boundedness and compactness of general Toeplitz operators on the $\alpha$-Bloch spaces.

Received by the editors October 29, 2004 and, in revised form, June 15, 2005.

2000 Mathematics Subject Classification. Primary 47B35; Secondary 32A18.

Key words and phrases. Toeplitz operators, $\alpha$-Bloch spaces.

The research of the first author was supported in part by NSF grant DMS 0200587.

The research of the third author was supported in part by an NSERC grant.

(C)2006 American Mathematical Society Reverts to public domain 28 years from publication 
For $\alpha>0$, the $\alpha$-Bloch spaces $B^{\alpha}$ are the spaces of analytic functions $f$ on $\mathbb{D}$ such that

$$
M^{\alpha}(f)=\sup _{z \in \mathbb{D}}\left|f^{\prime}(z)\right|\left(1-|z|^{2}\right)^{\alpha}<\infty .
$$

Each $B^{\alpha}$ is a Banach space with norm of $f$ equal to $\|f\|_{B^{\alpha}}=M^{\alpha}(f)+|f(0)|$.

An analytic function on $\mathbb{D}$ belongs to the little $\alpha$-Bloch space $B_{0}^{\alpha}, \alpha>0$, whenever

$$
\lim _{|z| \rightarrow 1}\left|f^{\prime}(z)\right|\left(1-|z|^{2}\right)^{\alpha}=0 .
$$

The spaces $B_{0}^{\alpha}$ are the subspaces of $B^{\alpha}$ that are the closure of the polynomials with respect to the $B^{\alpha}$ norm.

The $\alpha$-Bloch spaces and operators on them have been studied in many different contexts. For more references and details on the next few facts stated below see [9] and 10 .

For $\alpha=1, B^{1}=B$ is the classical Bloch space. There is a natural connection between the Bloch space and the Toeplitz operators via the fact that $P\left(L^{\infty}\right)=B$. A necessary condition for boundedness of $T_{f}$ on $B$ is that $P f \in B$, and so the condition is satisfied whenever $f \in L^{\infty}$. As we will see later, this is not a sufficient condition for boundedness of $T_{f}$ on $B$.

In general, the growth condition of a function $f$ in $B^{\alpha}$ with $f(0)=0$ is determined by $|f(z)| \leq\|f\|_{B^{\alpha}} \int_{0}^{1} \frac{|z| d t}{(1-|z| t)^{\alpha}}$. Thus, for $\alpha=1$, we get that

$$
|f(z)| \leq\|f\|_{B} \log \frac{1}{1-|z|}
$$

while for $\alpha>1$ we have that $|f(z)| \leq \frac{1}{\alpha-1}\|f\|_{B^{\alpha}} \frac{1}{(1-|z|)^{\alpha-1}}$. For $0<\alpha<1$, the spaces $B^{\alpha}$ are Lipschitz spaces $\operatorname{Lip}_{1-\alpha}$ and $B^{\alpha} \subset H^{\infty}$.

For $0<\alpha<\beta$, we have that $B^{\alpha} \subset B^{\beta}$. The Bloch space $B$ is included in all Bergman spaces $L_{a}^{p}, p \geq 1$, but for large $\alpha$, such as $\alpha \geq 2, B^{\alpha}$ gets so large that, for example, it includes the Bergman space $L_{a}^{2}$.

In presenting our results we will also refer to the logarithmic Bloch space.

The logarithmic Bloch space $L B$ is the space of analytic functions $f$ on $\mathbb{D}$ such that

$$
\sup _{z \in \mathbb{D}}\left|f^{\prime}(z)\right|\left(1-|z|^{2}\right) \log \frac{2}{1-|z|^{2}}<\infty .
$$

Correspondingly, the little logarithmic Bloch space $L B_{0}$ is the space of analytic functions $f$ on $\mathbb{D}$ such that

$$
\lim _{|z| \rightarrow 1}\left|f^{\prime}(z)\right|\left(1-|z|^{2}\right) \log \frac{2}{1-|z|^{2}}=0 .
$$

Throughout the paper, in order to have the operator $T_{\mu}^{\alpha}, \alpha>0$ well defined, we will assume that the complex measure $\mu$ is such that, for all $\mathrm{g}$ in $B^{\alpha}$

$$
\int_{\mathbb{D}}|g(w)|\left(1-|w|^{2}\right)^{\alpha-1}|d \mu(w)|<\infty .
$$

Note that in the case when $d \mu(z)=f(z) d A(z)$, a sufficient condition on $\mu$, so that the integral above is finite, is that $f \in L^{1}$, when $\alpha>1, f \in L^{1}\left(\log \frac{1}{1-|w|^{2}} d A(w)\right)$, when $\alpha=1$ and $f \in L^{1}\left(\left(1-|w|^{2}\right)^{\alpha-1} d A(w)\right)$, when $0<\alpha<1$. 


\section{Bounded TOEPlitz operators}

In this section we will present our main characterization of bounded Toeplitz operators on general $\alpha$-Bloch spaces. The result includes a restriction on the inducing measure that is a generalization of several known cases.

For a complex measure $\mu$ and $\alpha>0$, we will say that $\mu$ satisfies the condition $R_{\alpha}$ if

$$
R_{\alpha}(\mu)(w)=\alpha\left(1-|w|^{2}\right) \int_{\mathbb{D}} \frac{\left(1-|z|^{2}\right)^{\alpha-1}}{(\overline{z-w})(1-w \bar{z})^{\alpha+1}} d \mu(z) \in L^{\infty} .
$$

We get another form of $R_{\alpha}$ using the identity $\left(1-|w|^{2}\right)=1-w \bar{z}+w(\bar{z}-\bar{w})$ :

$$
R_{\alpha}(\mu)(w)=\alpha \int_{\mathbb{D}} \frac{\left(1-|z|^{2}\right)^{\alpha-1}}{(\overline{z-w})(1-w \bar{z})^{\alpha}} d \mu(z)+w P_{\alpha-1}(\mu)(w) .
$$

The special case when the measure $\mu$ is such that $d \mu(z)=f(z) d A(z)$, with $f \in H^{\infty}$, is the case when the Toeplitz operator is a multiplication operator. For $f \in B^{\alpha}$, as we will see below, $\mu$ satisfies the condition $R_{\alpha}$ if and only if $f \in H^{\infty}$.

Arazy's ([1]) and Zhu's ([8 and [10]) results provide a complete characterization of bounded multiplication operators on $\alpha$-Bloch spaces. We will state them in terms of multipliers.

Theorem A ([10]). Let $M(X)$ denote the space of multipliers of the Banach space of functions $X$, i.e., $M(X)=\{f \in X: f g \in X, \forall g \in X\}$.

(i) If $0<\alpha<1$, then $M\left(B^{\alpha}\right)=B^{\alpha}$ and $M\left(B_{0}^{\alpha}\right)=B_{0}^{\alpha}$.

(ii) If $\alpha=1$, then $M\left(B^{\alpha}\right)=M\left(B_{0}^{\alpha}\right)=H^{\infty}(\mathbb{D}) \cap L B$.

(iii) If $\alpha>1$, then $M\left(B^{\alpha}\right)=M\left(B_{0}^{\alpha}\right)=H^{\infty}(\mathbb{D})$.

As a consequence of the above theorem we can see that, for example, not every bounded function $f$ induces a bounded Toeplitz operator $T_{f}$ on the Bloch space $B$. As we will see later, for $f \in L^{\infty}, T_{f}$ is bounded on $B$ if and only if $P f \in L B$, which is a generalization of the analytic case of the above theorem. We will also see that there exist unbounded $L^{1}$ functions that induce bounded Toeplitz operators on the Bloch spaces.

In the next proposition we give more details about the condition $R_{\alpha}$ in some special cases.

Proposition 2.1. Let $\mu$ be a measure such that $d \mu(z)=f(z) d A(z)$, with $f \in L^{1}$. Then:

(i) If $f \in L^{\infty}, \mu$ satisfies the condition $R_{\alpha}, \alpha>0$.

(ii) If $f$ is analytic and in $B^{\alpha}$, then $R_{\alpha}(\mu)(w)=w f(w)$.

(iii) If $f$ is conjugate analytic, $\bar{f}$ is in $B^{\alpha}$ and $f(0)=0$, then $R_{\alpha}(\mu)(w)=$ $\frac{1}{\bar{w}} f(w)$.

Proof. Let $\psi_{w}(z)=\frac{w-z}{1-\bar{w} z}$, for $z, w$ in $\mathbb{D}$.

(i) For $\alpha>0$ and $f \in L^{\infty}$,

$$
\begin{aligned}
\left|R_{\alpha}(\mu)(w)\right| & =\alpha\left(1-|w|^{2}\right)\left|\int_{\mathbb{D}} \frac{\left(1-|z|^{2}\right)^{\alpha-1} \bar{f}(z)}{(z-w)(1-\bar{w} z)^{\alpha+1}} d A(z)\right| \\
& \leq \alpha\|f\|_{\infty}\left(1-|w|^{2}\right) \int_{\mathbb{D}} \frac{\left(1-|z|^{2}\right)^{\alpha-1}}{|z-w \| 1-\bar{w} z|^{\alpha+1}} d A(z) \\
& \leq c \alpha\|f\|_{\infty}\left(1-|w|^{2}\right)\left(1-|w|^{2}\right)^{-1}=c \alpha\|f\|_{\infty} .
\end{aligned}
$$


The second inequality follows from the fact that $\int_{\mathbb{D}} \frac{\left(1-|z|^{2}\right)^{\beta}}{|z-w||1-\bar{w} z|^{\gamma}} d A(z)$ is equivalent to $\left(1-|w|^{2}\right)^{\beta-\gamma+1}$, whenever $\beta-\gamma+1<0$, which can be easily derived from the well-known Forelli-Rudin estimates (see [6], page 17, or [8], page 53). The half-plane version of the proof of this equivalency can be found in [7].

Thus $R_{\alpha}(\mu)(w) \in L^{\infty}$, and (i) is proved.

(ii) Let $f$ be in $B^{\alpha}$. With a change of variable $z=\psi_{w}(u)$, we get that

$$
\begin{aligned}
R_{\alpha}(\mu)(w) & =\alpha \int_{\mathbb{D}} \frac{\left(1-\left|\psi_{w}(u)\right|^{2}\right)^{\alpha-1}\left|\psi_{w}^{\prime}(u)\right|^{2}}{-\bar{u}\left(1-w \bar{\psi}_{w}(u)\right)^{\alpha+2}} f \circ \psi_{w}(u) d A(u)+w P_{\alpha-1}(f)(w) \\
& =-\alpha \int_{\mathbb{D}} \frac{\left(1-|u|^{2}\right)^{\alpha-1}}{\bar{u}(1-\bar{w} u)^{\alpha+1}} f \circ \psi_{w}(u) d A(u)+w f(w)=I+w f(w) .
\end{aligned}
$$

For a fixed $w$ we have that $\frac{f \circ \psi_{w}(u)}{(1-\bar{w} u)^{\alpha+1}}$ belongs to $B^{\alpha}$ whenever $f \in B^{\alpha}$, and so $\frac{\left(1-|u|^{2}\right)^{\alpha-1} f \circ \psi_{w}(u)}{(1-\bar{w} u)^{\alpha+1}}$ must be in $L^{1}$. Using Taylor series expansion it is not hard to see that then $I=0$. Hence $R_{\alpha}(\mu)(w)=w f(w)$.

(iii) Let $f$ be conjugate analytic and let $f(0)=0$. Then $P_{\alpha-1}(f)(w)=0$, and as in the proof of part (ii), we have that

$$
\begin{aligned}
\overline{R_{\alpha}(\mu)(w)} & =-\alpha \int_{\mathbb{D}} \frac{\left(1-|u|^{2}\right)^{\alpha-1}}{u(1-\bar{u} w)^{\alpha+1}} \overline{f \circ \psi_{w}}(u) d A(u) \\
& =-\alpha \int_{\mathbb{D}} \frac{\left(1-|u|^{2}\right)^{\alpha-1}}{(1-\bar{u} w)^{\alpha+1}} \frac{\overline{f \circ \psi_{w}}(u)-\bar{f}(w)}{u} d A(u)
\end{aligned}
$$

since $\int_{\mathbb{D}} \frac{\left(1-|u|^{2}\right)^{\alpha-1}}{u(1-\bar{u} w)^{\alpha+1}} d A(u)=0$. Using the fact that $\frac{\overline{f \circ \psi_{w}}(u)-\bar{f}(w)}{u}$ is an analytic integrable function, we get that

$$
\overline{R_{\alpha}(\mu)(w)}=-\frac{\overline{f \circ \psi_{w}}(w)-\bar{f}(w)}{w}=-\frac{\bar{f}(0)-\bar{f}(w)}{w}=\frac{1}{w} \bar{f}(w) .
$$

Remark. If $f$ from the previous proposition is harmonic, then $R_{\alpha}(\mu)$ is also harmonic. If also $f$ is a harmonic extension of an $L^{1}(T)$ function, then $R_{\alpha}(\mu)\left(e^{i \theta}\right)=$ $e^{i \theta} f\left(e^{i \theta}\right)$. Thus, for $f$ harmonic and in $L^{\infty}$, we have that $R_{\alpha}(\mu)$ is also harmonic and in $L^{\infty}$, with $\left\|R_{\alpha}(\mu)\right\|_{\infty}=\|f\|_{\infty}$.

We will use the following lemma in the proof of our next theorem. The result is known in a more general form and can be found, for example, in [2]. We include a proof for completeness.

Lemma 2.1. Let $h \in L_{a}^{1}$ and $g \in B^{\alpha}, \alpha>0$. Then we have that

$$
H_{\bar{g}}^{\alpha} h(z)=\left(I-P_{\alpha}\right)(\bar{g} h)(z)=\int_{\mathbb{D}} \frac{\overline{g^{\prime}(w)} h(w)\left(1-|w|^{2}\right)^{\alpha+1}}{(z-w)(1-\bar{w} z)^{\alpha+1}} d A(w)
$$


Proof. We will use the following three formulas which can be found in [3] and [10]. For $g \in B^{\alpha}$ and $h \in L_{a}^{1}$ :
(a) $H_{\bar{g}}^{\alpha} h(z)=(\alpha+1) \int_{\mathbb{D}} \frac{(\bar{g}(z)-\bar{g}(u))\left(1-|u|^{2}\right)^{\alpha}}{(1-\bar{u} z)^{\alpha+2}} h(u) d A(u)$,
(b) $g(z)=g(0)+\int_{\mathbb{D}} \frac{\left(1-|u|^{2}\right)^{\alpha+1} g^{\prime}(u)}{\bar{u}(1-\bar{u} z)^{\alpha+2}} d A(u)$,
(c) $\left(\left(H_{\bar{g}}^{\alpha} h\right) \circ \psi_{z}\right)\left(\psi_{z}^{\prime}\right)^{\frac{\alpha+2}{2}}=H_{\bar{g} \circ \psi_{z}}^{\alpha}\left(\left(h \circ \psi_{z}\right)\left(\psi_{z}^{\prime}\right)^{\frac{\alpha+2}{2}}\right)$.

Without loss of generality, let us assume that $g(0)=0$. Then

$$
\begin{aligned}
& H_{\bar{g}}^{\alpha} h(0)=(\alpha+1) \int_{\mathbb{D}}(-\bar{g}(u))\left(1-|u|^{2}\right)^{\alpha} h(u) d A(u) \\
& =-(\alpha+1) \int_{\mathbb{D}} \overline{\left(\int_{\mathbb{D}} \frac{\left(1-|w|^{2}\right)^{\alpha+1} g^{\prime}(w)}{\bar{w}(1-\bar{w} u)^{\alpha+2}} d A(w)\right)}\left(1-|u|^{2}\right)^{\alpha} h(u) d A(u) \\
& =-(\alpha+1) \int_{\mathbb{D}} \frac{\left(1-|w|^{2}\right)^{\alpha+1} \overline{g^{\prime}}(w)}{w}\left(\int_{\mathbb{D}} \frac{\left(1-|u|^{2}\right)^{\alpha} h(u)}{(1-\bar{u} w)^{\alpha+2}} d A(u)\right) d A(w) \\
& =-\int_{\mathbb{D}} \frac{\left(1-|w|^{2}\right)^{\alpha+1} \overline{g^{\prime}}(w)}{w} h(w) d A(w) .
\end{aligned}
$$

Since $1-\left|\psi_{z}(v)\right|^{2}=\left(1-|v|^{2}\right)\left|\psi_{z}^{\prime}(v)\right|$ and $\psi_{z}^{\prime}(v)=\frac{|z|^{2}-1}{(1-\bar{z} v)^{2}}=\frac{1}{\psi_{z}^{\prime}\left(\psi_{z}(v)\right)}$, we have:

$$
\begin{aligned}
H_{\bar{g}}^{\alpha} h(z) & =\frac{1}{\left(|z|^{2}-1\right)^{\frac{\alpha+2}{2}}}\left(\left(H_{\bar{g}}^{\alpha} h\right) \circ \psi_{z}\right)(0)\left(\psi_{z}^{\prime}(0)\right)^{\frac{\alpha+2}{2}} \\
& =\frac{1}{\left(|z|^{2}-1\right)^{\frac{\alpha+2}{2}}}\left(H_{\bar{g} \circ \psi_{z}}^{\alpha}\left(\left(h \circ \psi_{z}\right)\left(\psi_{z}^{\prime}\right)^{\frac{\alpha+2}{2}}\right)\right)(0) \\
& =\frac{-1}{\left(|z|^{2}-1\right)^{\frac{\alpha+2}{2}}} \int_{\mathbb{D}} \frac{\left(1-|w|^{2}\right)^{\alpha+1} \overline{\left(g \circ \psi_{z}\right)^{\prime}}(w)}{w} h \circ \psi_{z}(w)\left(\psi_{z}^{\prime}(w)\right)^{\frac{\alpha+2}{2}} d A(w) \\
& =-\int_{\mathbb{D}} \frac{\overline{g^{\prime}}(v) h(v)\left(1-|v|^{2}\right)^{\alpha+1}\left(1-|z|^{2}\right)(1-\bar{z} v)(1-\bar{v} z)^{2}}{(z-v)(1-\bar{v} z)^{\alpha+2}\left(|z|^{2}-1\right)|1-\bar{z} v|^{2}} d A(v) \\
& =\int_{\mathbb{D}} \frac{\bar{g}^{\prime}(v) h(v)\left(1-|v|^{2}\right)^{\alpha+1}}{(z-v)(1-\bar{v} z)^{\alpha+1}} d A(v) .
\end{aligned}
$$

We have the following theorem:

Theorem 2.1. Suppose $\mu$ satisfies the condition $R_{\alpha}$, i.e.,

$$
R_{\alpha}(\mu)(w)=\alpha\left(1-|w|^{2}\right) \int_{\mathbb{D}} \frac{\left(1-|z|^{2}\right)^{\alpha-1}}{(\overline{z-w})(1-w \bar{z})^{\alpha+1}} d \mu(z) \in L^{\infty} .
$$

Then we have:

(i) If $0<\alpha<1$, then $T_{\mu}^{\alpha}$ is bounded on $B^{\alpha}$ if and only if $P_{\alpha-1}(\mu) \in B^{\alpha}$.

(ii) If $\alpha=1$, then $T_{\mu}^{\alpha}$ is bounded on $B^{\alpha}$ if and only if $P_{\alpha-1}(\mu) \in L B$.

(iii) If $\alpha>1$, then $T_{\mu}^{\alpha}$ is bounded on $B^{\alpha}$ if and only if $P_{\alpha-1}(\mu) \in B$.

Proof. Let $g \in B^{\alpha}$ and $h \in L_{a}^{1}$. It is known that $\left(L_{a}^{1}\right)^{*}=B^{\alpha}$ under the integral pairing $\langle h, g\rangle_{\alpha}=\alpha \int_{\mathbb{D}} h(z) \bar{g}(z)\left(1-|z|^{2}\right)^{\alpha-1} d A(z)$. See [10, Theorem 14] for more details. 
Thus, by Fubini's Theorem,

$$
\begin{aligned}
\left\langle h, T_{\mu}^{\alpha}(g)\right\rangle_{\alpha}= & \alpha \int_{\mathbb{D}} h(z) \overline{T_{\mu}^{\alpha}(g)(z)}\left(1-|z|^{2}\right)^{\alpha-1} d A(z) \\
= & \alpha \int_{\mathbb{D}} h(z) \overline{g(z)}\left(1-|z|^{2}\right)^{\alpha-1} d \bar{\mu}(z) \\
= & \alpha \int_{\mathbb{D}} P_{\alpha}(h \bar{g})(z)\left(1-|z|^{2}\right)^{\alpha-1} d \bar{\mu}(z) \\
& \quad+\alpha \int_{\mathbb{D}}\left[\left(I-P_{\alpha}\right)(h \bar{g})\right](z)\left(1-|z|^{2}\right)^{\alpha-1} d \bar{\mu}(z)=I_{1}+I_{2} .
\end{aligned}
$$

From Lemma 2.1 we have that

$$
\left[\left(I-P_{\alpha}\right)(h \bar{g})\right](z)=\int_{\mathbb{D}} \frac{\overline{g^{\prime}(w)} h(w)\left(1-|w|^{2}\right)^{\alpha+1}}{(z-w)(1-\bar{w} z)^{\alpha+1}} d A(w) .
$$

Hence, by (1),

$$
\begin{aligned}
\left|I_{2}\right| & =\left|\alpha \int_{\mathbb{D}} \int_{\mathbb{D}} \frac{\overline{g^{\prime}(w)} h(w)\left(1-|w|^{2}\right)^{\alpha+1}}{(z-w)(1-\bar{w} z)^{\alpha+1}} d A(w)\left(1-|z|^{2}\right)^{\alpha-1} d \bar{\mu}(z)\right| \\
& =\left|\int_{\mathbb{D}} \overline{g^{\prime}(w)} h(w)\left(1-|w|^{2}\right)^{\alpha} \overline{R_{\alpha}(\mu)(w)} d A(w)\right| \\
& \leq\|g\|_{B^{\alpha}}\|h\|_{1}\left\|R_{\alpha}(\mu)\right\|_{\infty} \leq C<\infty .
\end{aligned}
$$

On the other hand, by Fubini's Theorem,

$$
I_{1}=\int_{\mathbb{D}} h(w) \overline{g(w) Q_{\alpha}(\mu)(w)}\left(1-|w|^{2}\right)^{\alpha} d A(w),
$$

where $Q_{\alpha}(\mu)(w)=\alpha(\alpha+1) \int_{\mathbb{D}} \frac{\left(1-|z|^{2}\right)^{\alpha-1}}{(1-\bar{z} w)^{\alpha+2}} d \mu(z)$.

Hence $T_{\mu}^{\alpha}(g) \in B^{\alpha}$ if and only if

$$
\left(1-|w|^{2}\right)^{\alpha} g(w) Q_{\alpha}(\mu)(w) \in L^{\infty} .
$$

The relation between $Q_{\alpha}(\mu)$ and $P_{\alpha-1}(\mu)$ is

$$
Q_{\alpha}(\mu)(w)=(\alpha+1) P_{\alpha-1}(\mu)(w)+w P_{\alpha-1}^{\prime}(\mu)(w) .
$$

It is easy to see that $P_{\alpha-1}(\mu)$ is in $B^{\alpha}, L B$ and $B$ as $0<\alpha<1, \alpha=1$ and $\alpha>1$, respectively, implies that $Q_{\alpha}(\mu)$ satisfies, respectively:

$$
\begin{aligned}
& Q_{\alpha}(\mu)(w)\left(1-|w|^{2}\right)^{\alpha} \in L^{\infty}, \text { if } 0<\alpha<1 ; \\
& Q_{\alpha}(\mu)(w)\left(1-|w|^{2}\right) \log \frac{2}{1-|w|^{2}} \in L^{\infty}, \text { if } \alpha=1 ; \\
& Q_{\alpha}(\mu)(w)\left(1-|w|^{2}\right) \in L^{\infty}, \text { if } \alpha>1 .
\end{aligned}
$$

Notice that $B^{\alpha} \subset H^{\infty}$ as $0<\alpha<1$ and using the fact that, as $\alpha>1, g \in B^{\alpha}$ if and only if $\left(1-|w|^{2}\right)^{\alpha-1} g(w) \in L^{\infty}$, we easily see that (2) is true for all these cases. Thus, $T_{\mu}^{\alpha}$ is bounded on $B^{\alpha}$.

Conversely, if $T_{\mu}^{\alpha}$ is bounded on $B^{\alpha}$, then $T_{\mu}^{\alpha}(1)=P_{\alpha-1}(\mu) \in B^{\alpha}$. If $R_{\alpha}(\mu) \in$ $L^{\infty}$, as above, we get that there is a constant $c>0$, independent of $g$, such that

$$
\left(1-|w|^{2}\right)^{\alpha}\left|g(w) Q_{\alpha}(\mu)(w)\right| \leq c\|g\|_{B^{\alpha}} .
$$

For $0<\alpha<1$, we have already shown that $P_{\alpha-1}(\mu) \in B^{\alpha}$. 
For $\alpha \geq 1$, using test functions $g_{a}(w)=\log \frac{2}{1-\bar{a} w}$ and $g_{a}(w)=(1-\bar{a} w)^{1-\alpha}$, as $\alpha=1$ and $\alpha>1$, respectively, we get

$$
\begin{aligned}
& Q_{\alpha}(\mu)(w)\left(1-|w|^{2}\right) \log \frac{2}{1-|w|^{2}} \in L^{\infty} \quad \text { if } \alpha=1 ; \\
& Q_{\alpha}(\mu)(w)\left(1-|w|^{2}\right) \in L^{\infty} \quad \text { if } \alpha>1 .
\end{aligned}
$$

If $\alpha=1, P_{\alpha-1}(\mu) \in B$ implies that $P_{\alpha-1}(\mu)(w)\left(1-|w|^{2}\right) \log \frac{2}{1-|w|^{2}} \in L^{\infty}$. Thus, using (3) and (4), we easily see that $P_{\alpha-1}(\mu)^{\prime}(w)\left(1-|w|^{2}\right) \log \frac{2}{1-|w|^{2}} \in L^{\infty}$.

If $\alpha>1$, then $P_{\alpha-1}(\mu) \in B^{\alpha}$ implies that

$$
P_{\alpha-1}(\mu)(w)\left(1-|w|^{2}\right)^{\alpha-1} \in L^{\infty} .
$$

For $\alpha-1 \leq 1$, this implies that $P_{\alpha-1}(\mu)(w)\left(1-|w|^{2}\right) \in L^{\infty}$, and using (3) and (5), we get that $P_{\alpha-1}(\mu) \in B$.

For $\alpha-1>1$, combining (3), (5) and (6) we get $P_{\alpha-1}(\mu)^{\prime}(w)\left(1-|w|^{2}\right)^{\alpha-1} \in L^{\infty}$, which is equivalent to

$$
P_{\alpha-1}(\mu)(w)\left(1-|w|^{2}\right)^{\alpha-2} \in L^{\infty} .
$$

If $\alpha-2>1$, combining (3), (5) and (7) we get that $P_{\alpha-1}(\mu)^{\prime}(w)\left(1-|w|^{2}\right)^{\alpha-2}$ is in $L^{\infty}$, and we continue the process from above until we reach a positive integer $n$ such that $\alpha-n \leq 1$ and $P_{\alpha-1}(\mu)^{\prime}(w)\left(1-|w|^{2}\right)^{\alpha-n} \in L^{\infty}$.

Then, $P_{\alpha-1}(\mu)^{\prime}(w)\left(1-|w|^{2}\right) \in L^{\infty}$, and so $P_{\alpha-1}(\mu) \in B$.

This completes the proof.

Note that in the case $d \mu(z)=f(z) d A(z)$, with $f \in B^{\alpha}$, it follows from part (ii) of Proposition 2.1 that $R_{\alpha}(\mu)(w)=w f(w)$. Thus $\mu$ satisfies the condition $R_{\alpha}$ if and only if $f \in H^{\infty}$.

Corollary 2.1. Let $f \in L^{\infty}$ and let $T_{f}^{\alpha}(g)=P_{\alpha-1}(f g)$ for $g \in B^{\alpha}$. Then we have:

(i) If $0<\alpha<1$, then $T_{f}^{\alpha}$ is bounded on $B^{\alpha}$ if and only if $P_{\alpha-1}(f) \in B^{\alpha}$.

(ii) If $\alpha=1$, then $T_{f}^{\alpha}$ is bounded on $B^{\alpha}$ if and only if $P_{\alpha-1}(f) \in L B$.

(iii) If $\alpha>1$, then $T_{f}^{\alpha}$ is bounded on $B^{\alpha}$ if and only if $P_{\alpha-1}(f) \in B$.

Proof. Follows from Proposition 2.1 and Theorem 2.1.

Since the Bloch space $B$ is the dual of $L_{a}^{1}$ and $T_{\mu}^{*}=T_{\bar{\mu}}$ under this duality, we get the following corollary. Similar results including a few other restrictions can be found in 4 and 8 .

Corollary 2.2. Let $\mu$ be a complex measure satisfying condition $R_{\alpha}$. Then $T_{\bar{\mu}}$ is bounded on $L_{a}^{1}$ if and only if $P(\mu) \in L B$.

Remark. The proof of Theorem 2.1 implies that the condition $R_{\alpha}$ in the theorem can be replaced by a more general condition involving Carleson measures.

Recall that for a positive Borel measure $\nu$ on $\mathbb{D}$ we say that $\nu$ is a Carleson measure on the Bergman space if there exists $c>0$ such that, for all $h \in L_{a}^{2}$,

$$
\int_{\mathbb{D}}|h(z)|^{2} d \nu(z) \leq c \int_{\mathbb{D}}|h(z)|^{2} d A(z) .
$$

Let $\nu_{\alpha}(\mu)$ be the positive measure defined by $d \nu_{\alpha}(\mu)(z)=\left|R_{\alpha}(\mu)(z)\right| d A(z)$.

If $\mu$ satisfies condition $R_{\alpha}$, then it is easy to see that $\nu_{\alpha}(\mu)$ is a Carleson measure. By inspecting the proof of Theorem 2.1 we can see that, whenever $R_{\alpha}(\mu)$ is in $L^{1}$, 
the statements (i), (ii) and (iii) of Theorem 2.1 are still true if the condition $R_{\alpha}$ is replaced by a weaker one, namely, by requiring only that the measure $\nu_{\alpha}(\mu)$ is a Carleson measure for the Bergman space.

Note that the measure $\nu_{\alpha}(\mu)$ is a Carleson measure for the Bergman space if and only if there exists $r>0$, such that

$$
\sup _{z \in \mathbb{D}} \frac{1}{|D(z, r)|} \int_{D(z, r)}\left|R_{\alpha}(\mu)(w)\right| d A(w)<\infty,
$$

where $D(z, r)$ denotes the hyperbolic disk with center $z$ and radius $r$. See 9 , Theorem 6.2.2] for details.

Next we give an example of an unbounded $L^{1}$ function that induces a bounded Toeplitz operator on $B^{\alpha}$. We present part of the calculations as a separate lemma that covers a wider class of functions.

Lemma 2.2. Let $f(z)=\sum_{n=0}^{\infty} a_{n}\left(1-|z|^{2}\right)^{n}$ be a function in $L^{1}\left(\left(1-|z|^{2}\right)^{\alpha-1} d A(z)\right)$, for $\alpha>0$, and such that $\sum_{n=0}^{\infty} \frac{\left|a_{n}\right|}{n+\alpha}$ is finite. Let $d_{\alpha}=\alpha \sum_{n=0}^{\infty} \frac{a_{n}}{n+\alpha}$ and let $F_{\alpha}(f)=\int_{\mathbb{D}} f(z)\left(1-|z|^{2}\right)^{\alpha-1} d A(z)$. Then, for $d \mu(z)=f(z) d A(z)$ we have

$$
R_{\alpha}(\mu)(w)=d_{\alpha} w-\frac{\alpha}{\bar{w}}\left(F_{\alpha}(f)-\frac{1}{\left(1-|w|^{2}\right)^{\alpha}} \int_{D_{w}} f(z)\left(1-|z|^{2}\right)^{\alpha-1} d A(z)\right),
$$

where $D_{w}=\{z:|w|<|z|<1\}$.

Proof. As in the proof of part (ii) of Proposition 2.1 we have that, for $w \neq 0$,

$$
R_{\alpha}(\mu)(w)=w P_{\alpha-1}(f)(w)-\alpha \int_{\mathbb{D}} \frac{\left(1-|u|^{2}\right)^{\alpha-1}}{\bar{u}(1-\bar{w} u)^{\alpha+1}} f \circ \psi_{w}(u) d A(u) .
$$

Using the given series expansion of $f$ and the identity $1-\left|\psi_{w}(u)\right|^{2}=\frac{\left(1-|w|^{2}\right)\left(1-|u|^{2}\right)}{|1-\bar{w} u|^{2}}$, we get that $P_{\alpha-1}(f)(w)=\alpha \sum_{n=0}^{\infty} \frac{a_{n}}{n+\alpha}=d_{\alpha}$, and that the above integral equals

$$
\int_{\mathbb{D}} \sum_{n=0}^{\infty} a_{n}\left(1-|w|^{2}\right)^{n} \frac{\left(1-|u|^{2}\right)^{n+\alpha-1}}{(1-\bar{w} u)^{n+\alpha+1}} \frac{(1-\bar{u} w)^{-n}}{\bar{u}} d A(u) .
$$

Since $\frac{(1-\bar{u} w)^{-n}-1}{\bar{u}}$ is a conjugate analytic, integrable function, we have that

$$
(n+\alpha) \int_{\mathbb{D}} \frac{(1-\bar{u} w)^{-n}-1}{\bar{u}} \frac{\left(1-|u|^{2}\right)^{n+\alpha-1}}{(1-\bar{w} u)^{n+\alpha+1}} d A(u)=\frac{\left(1-|w|^{2}\right)^{-n}-1}{\bar{u}} .
$$

Since also $\int_{\mathbb{D}} \frac{\left(1-|u|^{2}\right)^{n+\alpha-1}}{\bar{u}(1-\bar{w} u)^{n+\alpha+1}} d A(u)=0$, we have that, furthermore,

$$
\begin{aligned}
R_{\alpha}(\mu)(w) & =d_{\alpha} w-\alpha \sum_{n=1}^{\infty} \frac{a_{n}}{n+\alpha}\left(1-|w|^{2}\right)^{n} \frac{\left(1-|w|^{2}\right)^{-n}-1}{\bar{w}} \\
& =d_{\alpha} w-\frac{\alpha}{\bar{w}}\left(\sum_{n=0}^{\infty} \frac{a_{n}}{n+\alpha}-\sum_{n=0}^{\infty} \frac{a_{n}}{n+\alpha}\left(1-|w|^{2}\right)^{n}\right) \\
& =d_{\alpha} w-\frac{\alpha}{\bar{w}}\left(F_{\alpha}(f)-\frac{1}{\left(1-|w|^{2}\right)^{\alpha}} \int_{D_{w}} f(z)\left(1-|z|^{2}\right)^{\alpha-1} d A(z)\right) .
\end{aligned}
$$


Example. For $\alpha>0$, let $c_{\alpha}=\frac{1}{\alpha}\left(\sum_{n=1}^{\infty} \frac{1}{n(n+\alpha)}\right)^{-1}$ and for $z \neq 0$, let

$$
f_{\alpha}(z)=1-c_{\alpha} \sum_{n=1}^{\infty} \frac{1}{n}\left(1-|z|^{2}\right)^{n}=1+c_{\alpha} \log \left(|z|^{2}\right)
$$

The function $f_{\alpha}$ is a radial, unbounded, $L^{1}$ function. Since $P_{\alpha-1}\left(f_{\alpha}\right)$ is a radial analytic function, it must be a constant. It is easy to check that $P_{\alpha-1}\left(f_{\alpha}\right)(0)=0$ and so $P_{\alpha-1}\left(f_{\alpha}\right)=0$.

In order to show that $T_{f_{\alpha}}$ is bounded on $B^{\alpha}$ we only have to prove that $\mu_{\alpha}$, with $d \mu_{\alpha}(z)=f_{\alpha}(z) d A(z)$, satisfies the condition $R_{\alpha}$. Using Lemma 2.2 and the fact that $d_{\alpha}=0$, we get that

$$
R_{\alpha}\left(\mu_{\alpha}\right)(w)=\frac{1}{\bar{w}}\left(1+\alpha c_{\alpha} \frac{1}{\left(1-|w|^{2}\right)^{\alpha}} \int_{|w|^{2}}^{1}(1-t)^{\alpha-1} \log t d t\right)
$$

It is easy to see that $\lim _{|w| \rightarrow 1}\left|R_{\alpha}(\mu)(w)\right|=1$. Since $\int_{0}^{1}(1-t)^{\alpha-1} \log t d t=\frac{-1}{\alpha c_{\alpha}}$, we also have that $\lim _{|w| \rightarrow 0}\left|R_{\alpha}(\mu)(w)\right|=\alpha$. Thus, $R_{\alpha}\left(\mu_{\alpha}\right) \in L^{\infty}$, and by Theorem 2.1, $T_{f_{\alpha}}$ is bounded on $B^{\alpha}$.

Note that we can also use Lemma 2.2 to show that $R_{\alpha}(\mu) \in L^{\infty}$ is not a necessary condition for the boundedness of the Toeplitz operator $T_{\mu}$. For example, let $d \mu(z)=$ $f(z) d A(z)$ with $f(z)=\frac{1}{|z|^{3 / 2}}$. Note that for this radial, positive function $F_{\alpha}(f)$ is equal to $\frac{\Gamma(1 / 4) \Gamma(\alpha)}{\Gamma(\alpha+1 / 4)}$, and $d_{\alpha}$ is equivalent to $\alpha \sum_{n=1}^{\infty} \frac{1}{n^{1 / 4}(n+\alpha)}$. Using Lemma 2.2 we can get that $\left|R_{\alpha}(\mu)(w)\right| \geq \frac{c}{|w|^{\frac{1}{2}}}$ and so $R_{\alpha}(\mu)$ is not in $L^{\infty}$. Since the measure $\nu_{\alpha}(\mu)$ is a Carleson measure, $T_{\mu}$ is still bounded on $B^{\alpha}$.

\section{Compact Toeplitz operators}

For the next result, we need the following lemma from [5].

Lemma 3.1. Let $0<\alpha<1$ and let $T$ be a bounded linear operator from $B^{\alpha}$ into a normed linear space $Y$. Then $T$ is compact if and only if $\left\|T g_{n}\right\|_{Y} \rightarrow 0$, whenever $\left(g_{n}\right)$ is a bounded sequence in $B^{\alpha}$ that converges to 0 uniformly on $\overline{\mathbb{D}}$.

Theorem 3.1. Suppose

$$
\lim _{|w| \rightarrow 1} R_{\alpha}(\mu)(w)=0
$$

Then we have:

(i) If $0<\alpha<1$, then $T_{\mu}^{\alpha}$ is compact on $B^{\alpha}$ if and only if $P_{\alpha-1}(\mu) \in B^{\alpha}$.

(ii) If $\alpha=1$, then $T_{\mu}^{\alpha}$ is compact on $B^{\alpha}$ if and only if $P_{\alpha-1}(\mu) \in L B_{0}$.

(iii) If $\alpha>1$, then $T_{\mu}^{\alpha}$ is compact on $B^{\alpha}$ if and only if $P_{\alpha-1}(\mu) \in B_{0}$.

Proof. For the case $\alpha=1$ or $\alpha>1$, let $\left\{g_{n}\right\}$ be a sequence in $B^{\alpha}$ such that $\left\|g_{n}\right\|_{B^{\alpha}} \leq 1$ and $g_{n}(z) \rightarrow 0$ uniformly on compact subsets of $\mathbb{D}$. Let $h$ be in the 
unit ball of $L_{a}^{1}$. Similarly, as in the proof of Theorem 2.1, we have

$$
\begin{aligned}
& \left\langle h, T_{\mu}^{\alpha}\left(g_{n}\right)\right\rangle_{\alpha} \\
& =\alpha \int_{\mathbb{D}} P_{\alpha}\left(h \bar{g}_{n}\right)(z)\left(1-|z|^{2}\right)^{\alpha-1} d \bar{\mu}(z) \\
& \quad+\alpha \int_{\mathbb{D}}\left[\left(I-P_{\alpha}\right)\left(h \bar{g}_{n}\right)\right](z)\left(1-|z|^{2}\right)^{\alpha-1} d \bar{\mu}(z) \\
& =I_{1}+I_{2} .
\end{aligned}
$$

For $0<r<1$, and $\mathbb{D}_{r}=\{z:|z| \leq r\}$,

$$
\begin{aligned}
I_{2} & =\alpha \int_{\mathbb{D}} \overline{g_{n}^{\prime}(w)} h(w)\left(1-|w|^{2}\right)^{\alpha} R_{\alpha}(\mu)(w) d A(w) \\
& =\alpha\left(\int_{\mathbb{D}_{r}}+\int_{\mathbb{D} \backslash \mathbb{D}_{r}}\right) \overline{g_{n}^{\prime}(w)} h(w)\left(1-|w|^{2}\right)^{\alpha} R_{\alpha}(\mu)(w) d A(w)=K_{1}+K_{2} .
\end{aligned}
$$

For a fixed $\varepsilon>0$, using condition (8), let $r$ be sufficiently close to 1 so that $\left|R_{\alpha}(\mu)(w)\right|<\varepsilon$ as $w \in \mathbb{D} \backslash \mathbb{D}_{r}$. Then $\left|K_{2}\right| \leq \varepsilon\left\|g_{n}\right\|_{B^{\alpha}}\|h\|_{1} \leq \varepsilon$.

Since $g_{n}(z) \rightarrow 0$ as $n \rightarrow \infty$, we can choose $n$ big enough so that $\left|g_{n}(z)\right|\left(1-|w|^{2}\right)^{\alpha}<\varepsilon$. Therefore, $\left|K_{1}\right| \leq \varepsilon\left\|R_{\alpha}(\mu)\right\|_{\infty}\|h\|_{1} \leq \varepsilon\left\|R_{\alpha}(\mu)\right\|_{\infty}$.

Hence $\left|I_{2}\right|<C \varepsilon$, where $C$ does not depend on $h$, and so $\lim _{n \rightarrow \infty} \sup _{\|h\|_{1} \leq 1}\left|I_{2}\right|$ $=0$. Thus $T_{\mu}^{\alpha}$ is compact on $B^{\alpha}$ if and only if $\sup _{\|h\|_{1} \leq 1}\left|I_{1}\right| \rightarrow 0$ as $n \rightarrow \infty$.

Similarly, as in the proof of Theorem 2.1, we have

$$
\begin{aligned}
I_{1} & =\int_{\mathbb{D}} h(w) \overline{g_{n}(w) Q_{\alpha}(\mu)(w)}\left(1-|w|^{2}\right)^{\alpha} d A(w) \\
& =\left(\int_{\mathbb{D}_{r}}+\int_{\mathbb{D} \backslash \mathbb{D}_{r}}\right) h(w) \overline{g_{n}(w) Q_{\alpha}(\mu)(w)}\left(1-|w|^{2}\right)^{\alpha} d A(w)=M_{1}+M_{2} .
\end{aligned}
$$

It is easy to see that if $P_{\alpha-1}(\mu)$ is in $L B_{0}$ and $B_{0}$ as $\alpha=1$ and $\alpha>1$, respectively, then, as $|w| \rightarrow 1, Q_{\alpha}$ satisfies, respectively:

$$
\begin{aligned}
& Q_{\alpha}(\mu)(w)\left(1-|w|^{2}\right) \log \frac{2}{1-|w|^{2}} \rightarrow 0, \text { if } \alpha=1 ; \\
& Q_{\alpha}(\mu)(w)\left(1-|w|^{2}\right) \rightarrow 0, \text { if } \alpha>1 .
\end{aligned}
$$

Again, notice that, as $\alpha>1, g \in B^{\alpha}$ if and only if $\left(1-|z|^{2}\right)^{\alpha-1} g(z) \in L^{\infty}$, and we may choose $r$ sufficiently close to 1 such that whenever $w \in \mathbb{D} \backslash \mathbb{D}_{r}$, $\left|Q_{\alpha}(\mu)(w)\right|\left(1-|w|^{2}\right) \log \frac{2}{1-|w|^{2}}<\varepsilon$ as $\alpha=1$; and $\left|Q_{\alpha}(\mu)(w)\right|\left(1-|w|^{2}\right)^{\alpha}<\varepsilon$ as $\alpha>1$, respectively.

We see that $\left|M_{2}\right| \leq \varepsilon \alpha\left\|g_{n}\right\|_{B^{\alpha}}\|h\|_{1} \leq C \varepsilon$, where $C$ does not depend on $h$.

Since $g_{n}(z) \rightarrow 0$ uniformly on compact subsets of $\mathbb{D}$, we can choose $n$ big enough so that $\left|g_{n}(w)\right|<\varepsilon$. Hence we easily see that, as $n$ is big enough, $\left|M_{1}\right|<C \varepsilon$, where $C$ does not depend on $h$. Therefore, $\sup _{\|h\|_{1} \leq 1}\left|I_{1}\right| \rightarrow 0$ as $n \rightarrow \infty$, and so $T_{\mu}^{\alpha}$ is compact on $B^{\alpha}$ as $\alpha=1$ or $\alpha>1$.

Now let $0<\alpha<1$. Let $\left\{g_{n}\right\}$ be a sequence in $B^{\alpha}$ such that $\left\|g_{n}\right\|_{B^{\alpha}} \leq 1$ and $g_{n}(z) \rightarrow 0$ uniformly on $\mathbb{D}$. Let $h$ be in the unit ball of $L_{a}^{1}$. By a similar discussion as above, $T_{\mu}^{\alpha}$ is compact on $B^{\alpha}$ if and only if $\sup _{\|h\|_{1} \leq 1}\left|I_{1}\right| \rightarrow 0$ as $n \rightarrow \infty$. However, 
since $\left\|g_{n}\right\|_{B^{\alpha}} \leq 1$ and $g_{n}(z) \rightarrow 0$ uniformly on $\overline{\mathbb{D}}$, we can choose $n$ big enough such that $\left|g_{n}(w)\right|<\varepsilon$ uniformly for $w \in \overline{\mathbb{D}}$.

Thus, as $n$ is big enough, $\left|I_{1}\right| \leq \varepsilon \alpha\left\|P_{\alpha-1}(\mu)\right\|_{B^{\alpha}}\|h\|_{1}$.

Therefore, $\sup _{\|h\|_{1} \leq 1}\left|I_{1}\right| \rightarrow 0$ as $n \rightarrow \infty$, and so $T_{\mu}^{\alpha}$ is compact on $B^{\alpha}$.

Conversely, if $T_{\mu}^{\alpha}$ is compact on $B^{\alpha}$. Suppose first that $\alpha=1$ or $\alpha>1$. Then $\sup _{\|h\|_{1} \leq 1}\left|I_{1}\right| \rightarrow 0$ as $n \rightarrow \infty$ for any sequence $g_{n}$ in $B^{\alpha}$ such that $\left\|g_{n}\right\|_{B^{\alpha}} \leq 1$ and $g_{n}(z) \rightarrow 0$ uniformly on compact subsets of $\mathbb{D}$.

Let $h_{z}(w)=\frac{\left(1-|z|^{2}\right)^{\alpha}}{(1-\bar{z} w)^{2+\alpha}}$. Then $\left\|h_{z}\right\|_{1} \leq C$, and

$$
\begin{aligned}
I_{1} & =\left(1-|z|^{2}\right)^{\alpha} \int_{\mathbb{D}} \frac{\overline{g_{n}(w) Q_{\alpha}(\mu)(w)}\left(1-|w|^{2}\right)^{\alpha}}{(1-\bar{z} w)^{2+\alpha}} d A(w) \\
& =(\alpha+1)^{-1}\left(1-|z|^{2}\right)^{\alpha} \frac{g_{n}(z) Q_{\alpha}(\mu)(z)}{}
\end{aligned}
$$

Thus, $\sup _{\|h\|_{1} \leq 1}\left|I_{1}\right| \rightarrow 0$ as $n \rightarrow \infty$ implies

$$
\lim _{n \rightarrow \infty} \sup _{z \in \mathbb{D}}\left(1-|z|^{2}\right)^{\alpha}\left|g_{n}(z)\right|\left|Q_{\alpha}(\mu)(z)\right|=0 .
$$

As $\alpha=1$ or $\alpha>1$, using testing functions $g_{a}(z)=\left(\log \frac{1}{1-|a|^{2}}\right)^{-1}\left(\log \frac{2}{1-\bar{a} z}\right)^{2}$ and $g_{a}(z)=\left(1-|a|^{2}\right)(1-\bar{a} z)^{-\alpha}$ respectively in (9), as in the proof of Theorem 2.1, we easily see that $P_{\alpha-1}(\mu)$ is in $L B_{0}$ and $B_{0}$, respectively.

Now let $0<\alpha<1$ and let $T_{\mu}^{\alpha}$ be compact on $B^{\alpha}$. Then $T_{\mu}^{\alpha}$ is bounded on $B^{\alpha}$. By Theorem 2.1, $P_{\alpha-1}(\mu) \in B^{\alpha}$. The proof is completed.

Remark. The requirement that $\lim _{|w| \rightarrow 1} R_{\alpha}(\mu)(w)=0$, in the proof of Theorem 3.1 , can be replaced by a more general requirement that the measure $\nu_{\alpha}(\mu)$, defined in Section 2, is a compact Carleson measure.

Recall that for a positive Borel measure $\nu$ on $\mathbb{D}$ we say that $\nu$ is a compact Carleson measure whenever

$$
\lim _{|z| \rightarrow 1} \frac{1}{|D(z, r)|} \int_{D(z, r)} d \nu(w)=0,
$$

where $D(z, r)$ denotes the hyperbolic disk with center $z$ and radius $r$. See 9 , Theorem 6.2.5] for more details. If the measure $\mu$ is such that $\lim _{|w| \rightarrow 1} R_{\alpha}(\mu)(w)=$ 0 , then it is easy to see that $\nu_{\alpha}(\mu)$ is a compact Carleson measure.

Note that in the case $d \mu(z)=f(z) d A(z)$, with $f \in B^{\alpha}$, it follows from part (ii) of Proposition 2.1 that $R_{\alpha}(\mu)(w)=w f(w)$. Thus the condition (8) in Theorem 3.1 is satisfied only when $f=0$.

\section{REFERENCES}

[1] J. Arazy, Multipliers of Bloch Functions, University of Haifa Mathem. Public. Series 54 (1982).

[2] J. Arazy, S. Fisher, S. Janson, J. Peetre, An Identity for Reproducing Kernels in a Planar Domain and Hilbert-Schmidt Hankel Operators, J. Reine Angew. Math. 406 (1990), 179-199.

[3] J. Arazy, S. Fisher, J. Peetre, Hankel Operators on Weighted Bergman Spaces, American J. Math. 110 (1988), 989-1054. MR0970119 (90a:47067)

[4] K. R. M. Attele, Toeplitz and Hankel Operators on Bergman One Space, Hokkaido Math. J. 21 (1992), 279-293. MR1169795 (93d:47051)

[5] S. Ohno, K. Stroethoff, R. Zhao, Weighted Composition Operators between Bloch-Type spaces, Rocky Mountain J. Math. 33 (2003), 191-215. MR1994487(2004d:47058) 
[6] W. Rudin, Function Theory in the Unit Ball in $\mathbb{C}^{n}$, Springer-Verlag, New York, 1980. MR0601594 (82i:32002)

[7] J. L. Wang, Z. Wu, Minimum solutions of $\bar{\delta}^{k+1}$ and Middle Hankel Operators, J. Funct. Anal. 118 (1993), 167-187. MR1245601 (94j:47042)

[8] K. Zhu, Multipliers of BMO in the Bergman Metric with Applications to Toeplitz Operators, J. Funct. Anal. 87 (1989), 31-50. MR:1025882 (90m:42024)

[9] K. Zhu, Operator Theory on Function Spaces, Marcel Dekker, New York, 1990. MR:1074007 (92c:47031)

[10] K. Zhu, Bloch-Type Spaces of Analytic Functions, Rocky Mountain J. Math. 23 (1993), 11431177. MR1245472 (95d:46020)

Department of Mathematics, University of Alabama, Tuscaloosa, Alabama 35487

E-mail address: zwu@gp.as.ua.edu

Department of Mathematics, University of Toledo, Toledo, Ohio 43606

E-mail address: Ruhan.Zhao@utoledo.edu

Current address: Department of Mathematics, SUNY-Brockport, Brockport, New York 14420

E-mail address: rzhao@brockport.edu

Department of Mathematics, University of Manitoba, Winnipeg, Manitoba, Canada R3T 2N2

E-mail address: zorbosk@cc.umanitoba.ca 